\title{
A Study of Causes And Rate of Neonatal Mortality in A Teritiary Care Teaching Hospital
}

\author{
Surender Kagitapu ${ }^{1}$, Madhuri Alapati ${ }^{2}$, Sneha Baira $^{2}$, Mounika Pilli ${ }^{2}$, Pavani B ${ }^{2}$, \\ Anusha $\mathrm{A}^{2}$ \\ Madhuri Alapati and Sneha Baira have equal contribution in work and considered as second authors \\ ${ }^{I}$ Department of Pediatrics, Kakatiya Medical College, Mahathma Gandhi Memorial Hospital, Warangal, \\ Telangana, India-506002. \\ ${ }^{2}$ Department of Clinical Pharmacy, Vaagdevi College of Pharmacy, Hanamkonda, Warangal, Telangana, India.
}

\begin{abstract}
The first 28 days of life is known as neonatal period. Neonatal death is defined as the death of live birth during first 28 days of life. It is highly vulnerable period that the infants are susceptible to certain problems which lead to illness and death. About 40\% of the 10 million deaths among children below 5 years occur during the neonatal period; this is twice as high as the number of deaths attributable to HIV/AIDS. The study was conducted between March 2014 and August 2014. It is a prospective observational study. Both male and female were included. All live neonates admitted to Neonatal Care Unit, Mahatma Gandhi Memorial Hospital were included in the study. Unknown babies also included in the study. Died neonates who brought to Mahatma Gandhi Memorial Hospital were excluded from the study. Cesarean deliveries in early term are more when compared to Normal and Assisted vaginal deliveries in early term. Major causes of deaths include LBW (62.16\%) followed by sepsis (54.59\%), RD (51.89\%), HIE (22.16\%) and Congenital abnormalities (15.13\%). In LBW major causes of death include Sepsis (87.8\%) followed by $R D(83.5 \%)$ and preterm deaths (87.8\%) are more when compared to term gestation (12.27\%). Further much work is needed to educate community members about neonatal danger signs and to engage them in effective care-seeking behavior.The process of delivery should be made safe for the mother and neonate. We found $L B W$, Sepsis and RD to be the major cause's neonatal death.
\end{abstract}

Keyword: Mortality, Low Birth weight, Sepsis, Respiratory distress

\section{Introduction}

The first 28 days of life is known as neonatal period. Neonatal death is defined as the death of live birth during first 28 days of life. It is highly vulnerable period that the infants are susceptible to certain problems which lead to illness and death. About $40 \%$ of the 10 million deaths among children below 5 years occur during the neonatal period; this is twice as high as the number of deaths attributable to HIV/AIDS. Almost $99 \%$ of neonatal deaths occur in developing countries and about two-third occurring in Africa and Southeast Asia ${ }^{[1]}$. Every year there is an estimated 3.1 million neonatal deaths occurring world wide ${ }^{[2]}$. The vast majority (up to $98.5 \%$ ) of neonatal deaths occur in developing countries ${ }^{[3]}$. In India, neonatal mortalities declining sluggishly and the statistics were in 2001 (40 per 1000 live births) in 2007 (36 per 1000 live births) and in 2010 (32 per 1000 live births). Within the neonatal period, $80 \%$ of all new born deaths occur during first week of life, it is called early neonatal period ${ }^{[4]}$. Neonatal mortality in United States in 2005 declined largely due to improvement in obstetric and neonatal intensive care as well as advances in diagnosis and treatment. Neonatal morbidity and mortality is still high in developing countries that are primarily due to negligence of female health, nutrition deliveries by unskilled personnel and poor antenatal care. In a global perspective the major causes of neonatal death are found to be prematurity, low birth weight, birth asphyxia and neonatal infections ${ }^{[5]}$. In 2005 about quarter of global neonatal deaths occur in India, which has a neonatal mortality of 43 per 1000 live births ${ }^{[6]}$, Whereas in Bangladesh high maternal and neonatal mortality rates are high at 194 per 10,000 live births and 37 per 1000 live births ${ }^{[7]}$. Most deaths in India, including of children, are not medically certified since most occur at home, in rural areas, and without attention by a health-care worker. Understanding of the causes of child death might be helpful to guide the use of widely practicable interventions for neonatal and child survival ${ }^{[8]}$. The proportion of early neonatal mortality (deaths in the first week of life) has increased as compared to the late neonatal mortality (deaths from 7 to 28 days of life) and it accounts for three quarters of all neonatal deaths. Among the early neonatal deaths, two thirds occur in the first 24 hours (very early neonatal mortality) ${ }^{[9]}$. In the ASIAN region, it is estimated that preterm birth complications account for (45\%), birth asphyxia for (25\%), congenital anomalies for (16\%), and sepsis or pneumonia for $(14 \%)$. These proportions vary among individual countries ${ }^{[10]}$. Low birth weight is an important cause of perinatal mortality and both short and long term infant and childhood morbidity. Low birth weight infants die at rates of up to 40 times those of infants of normal weight ${ }^{[11]}$. PRETERM BIRTH: Preterm birth is defined as babies born alive before 37 weeks of pregnancy. Preterm birth is a major determinant of 
neonatal mortality and morbidity which has long term adverse consequences on health ${ }^{[12]}$. Premature infants are at greater risk for short and long term complications, including disabilities and implements in growth and mental development ${ }^{[13]}$. Of all early neonatal deaths (deaths within the first 7 days of life) that are not associated with congenital malformations, $28 \%$ are due to preterm birth ${ }^{[14]}$. Preterm birth rates have been reported to range from $5 \%$ to $7 \%$ live births in some developed countries, but are estimated to be substantially higher in developing countries ${ }^{[15]}$. LOW BIRTH WEIGHT: Low birth weight (LBW) is defined as birth weight of a live born infant of less than 2,500g (5 pounds 8 ounces) regardless of gestational age ${ }^{[16]}$. Subcategories include Very Low Birth Weight (VLBW) which is less than 1500 gm, and Extremely Low Birth Weight (ELBW) which is less than 1000 $\mathrm{gm}^{[17]}$. Normal Weight at term delivery is $2500 \mathrm{gm}$ to $4200 \mathrm{gm}$.

\section{Materials And Materials}

MGM Hosital is a large government tertiary care teaching hospital located in Warangal District of Telangana State. It is a 1200 bed strength hospital with an area of 13 acres, which provides teaching facility to Kakatiya Medical College (KMC) students. The study was conducted between March 2014 and August 2014. It is a prospective observational study. Both male and female were included. All live neonates admitted to Neonatal Care Unit, Mahatma Gandhi Memorial Hospital were included in the study. Unknown babies also included in the study. Died neonates who brought to Mahatma Gandhi Memorial Hospital were excluded from the study.

\section{Results}

During the study period, a total of 1018 Neonates were admitted to SNCU. All cases were included in the study who met the study criteria. Out of 1018 total admissions 185 deaths were occurred and Neonatal Mortality Rate was found to be 18.172 per 100 live births (Table 1, Figure 1).

Table 1. Total number of admissions and deaths

\begin{tabular}{|c|c|}
\hline Total admissions & Total deaths \\
\hline 1018 & 185 \\
\hline
\end{tabular}

Figure1. Showing the total number of admissions and total number of deaths.

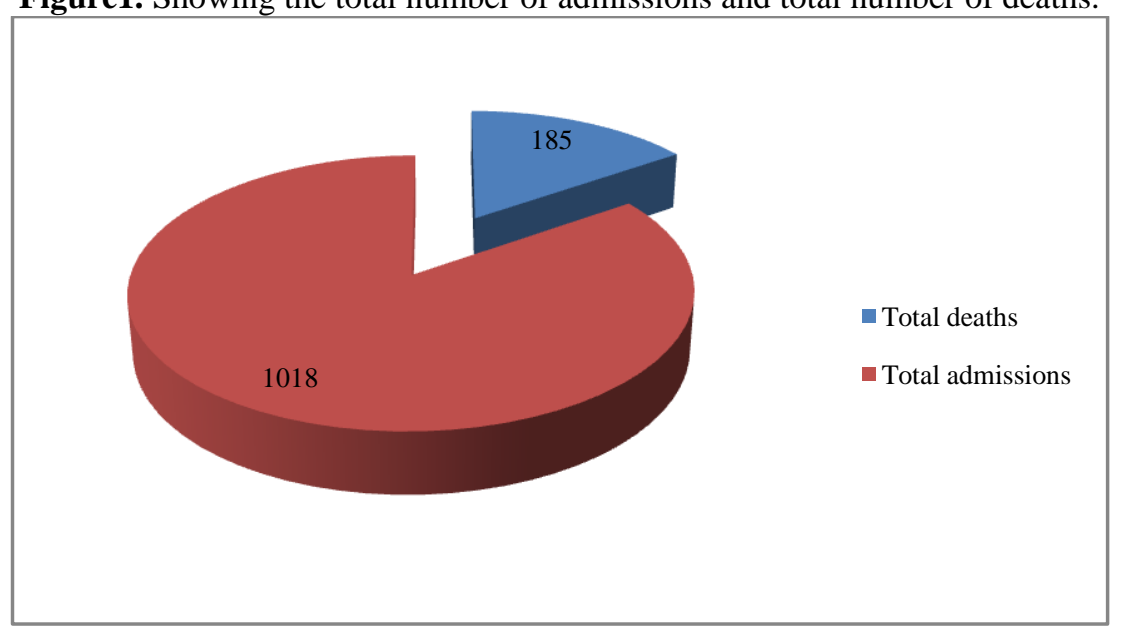

Out of all admissions 589 were males in which 111 (60\%) died and 429 were females in which $74(40 \%)$ died indicating that male neonatal deaths are more when compared to females $(\mathrm{RR}=1.093 ; \mathrm{CI}=0.8369-1.426 ; \mathrm{P}=$ 0.5647), (Table 5, Figure 2).

Table 2. Gender wise admissions and deaths

\begin{tabular}{|c|c|c|}
\hline Gender & Males & Females \\
\hline Total & 589 & 429 \\
\hline Deaths & 111 & $74 \mathrm{~s}$ \\
\hline
\end{tabular}


Figure 2. Showing the Gender wise admissions and deaths

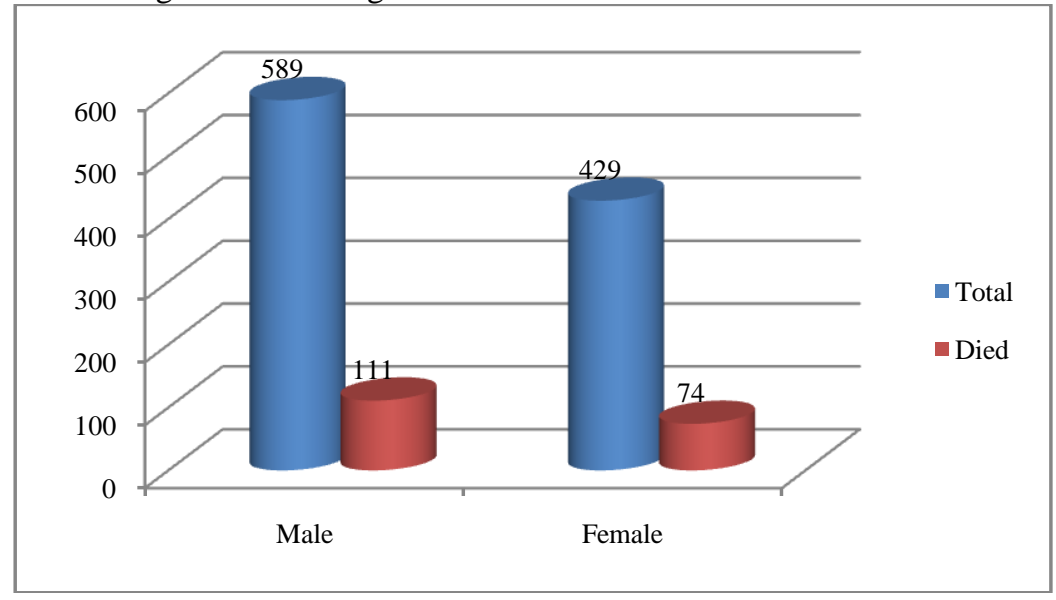

In our study most of admissions were from rural area and accounted for 802 in which $151(81.62 \%)$ babies died and 651 survived. From urban area total admissions were 216 out of which 34 (18.3\%) died and 182 survived (Table 3, Figure 3).

Table 3. Area wise Total admissions and deaths

\begin{tabular}{|c|c|c|}
\hline Neonates & Rural & Urban \\
\hline All births & 802 & 216 \\
\hline Deaths & 151 & 34 \\
\hline
\end{tabular}

Figure 3. Showing the Area wise Total admissions and deaths

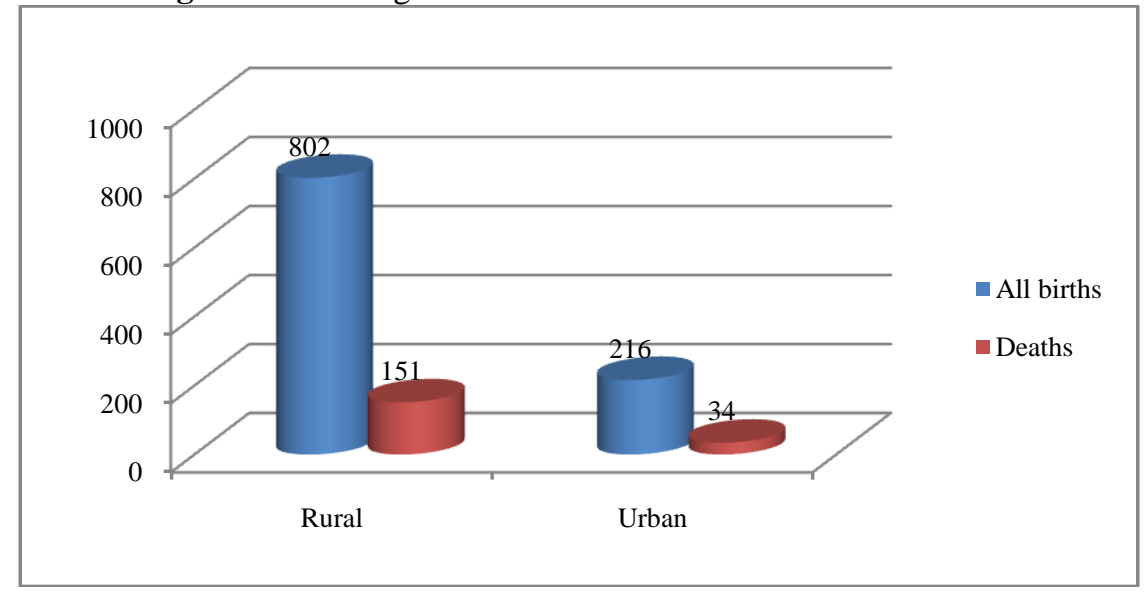

Table 4. Neonatal deaths according to area

\begin{tabular}{|c|c|c|c|c|c|c|}
\hline Area & All birth & Died & Survived & P value & Relative risk & $95 \%$ CI \\
\hline RURAL & & & & & & \\
YES & 802 & 151 & 651 & 0.3213 & 1.196 & $0.8510,1.081$ \\
NO & 216 & 34 & 182 & & & \\
\hline URBAN & 216 & 34 & 182 & 0.343 & 0.8360 & $0.5948,10175$ \\
YES & 802 & 151 & 651 & & \\
NO & & & & & \\
\hline
\end{tabular}

$13(7.02 \%)$ death out of 185 occurred during first day of life i.e. day $0,125(67.57 \%)$ death occurred during first week of life i.e. day 1-6. Collectively known as ear deaths. Late deaths at 7-27 days were 39 (21.08\%) (Table 8, figure 4).

Table 5. Timing of neonatal deaths

\begin{tabular}{|l|c|c|}
\hline Number of days & Total number of deaths & Death percentage \\
\hline $1^{\text {st }}$ day of life (i.e. Day 0) & 13 & $7.02 \%$ \\
\hline $1^{\text {st }}$ week of life (i.e.Day1-6) & 125 & $67.56 \%$ \\
\hline Late (7-27 Days) & 39 & $21.08 \%$ \\
\hline
\end{tabular}


Figure 4. Showing the Timing of neonatal deaths

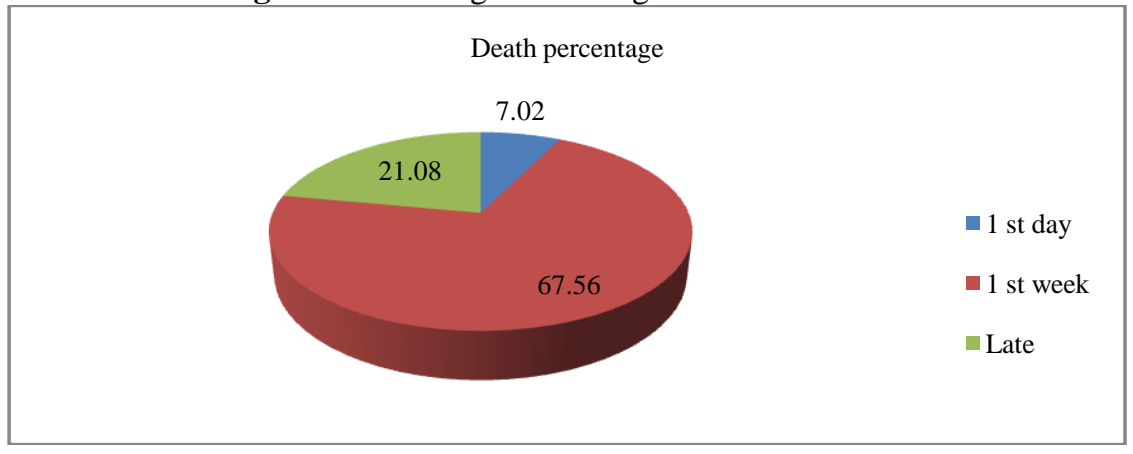

\section{Mode of delivery:}

535 Mothers has cesarean deliveries. And of these 91 neonatal deaths were noted. The major indications for cesarean delivery were Breech presentations, fetal distress, previous Cesarean delivery, absence of labor pains, Oligohydromnios cephalopelvic disproportions, polyhydromnios, twin gestation.

416 mothers had normal vaginal deliveries among which 81 neonatal deaths were noted 64 mothers had assisted vaginal deliveries of which 13 deaths were note (Table 6, Figure5).

Table 6. Deaths based on Mode of delivery

\begin{tabular}{|c|c|c|}
\hline Total & Cesarean & NVD+ AVD \\
\hline All births & 535 & 480 \\
\hline Deaths & 91 & 94 \\
\hline
\end{tabular}

Nvd: normal vaginal delivery

Avd: assisted vaginal delivery

Figure 5. Showing the Deaths based on Mode of delivery

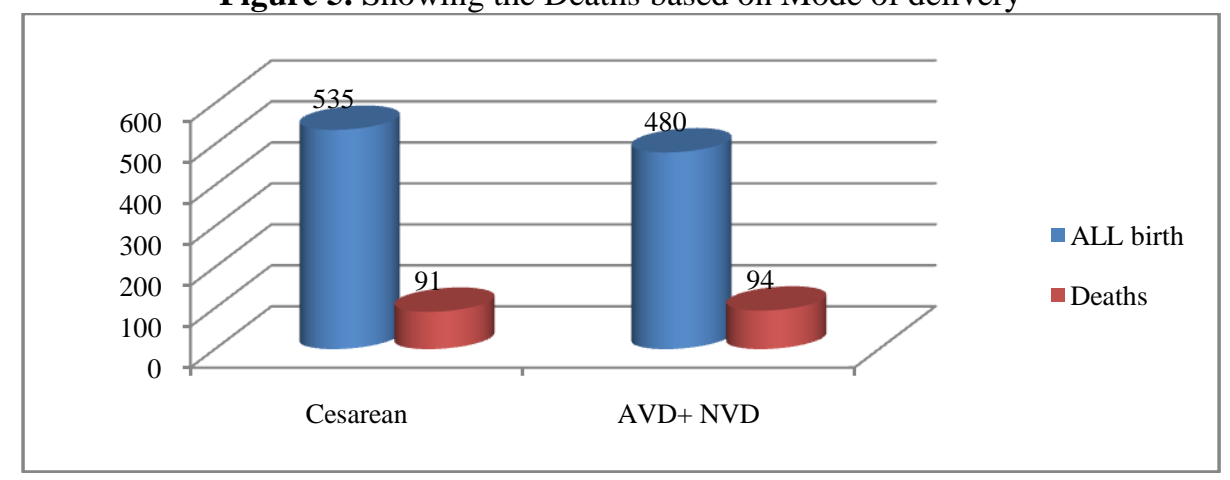

Table 7. Neonatal deaths in early term based on Mode of delivery

\begin{tabular}{|c|c|c|}
\hline Total & ET+ Cesarean & ET+ NVD \\
\hline All birth & 159 & 152 \\
\hline Died & 20 & 15 \\
\hline
\end{tabular}

Figure 6. Showing the Neonatal deaths in early term based on Mode of delivery

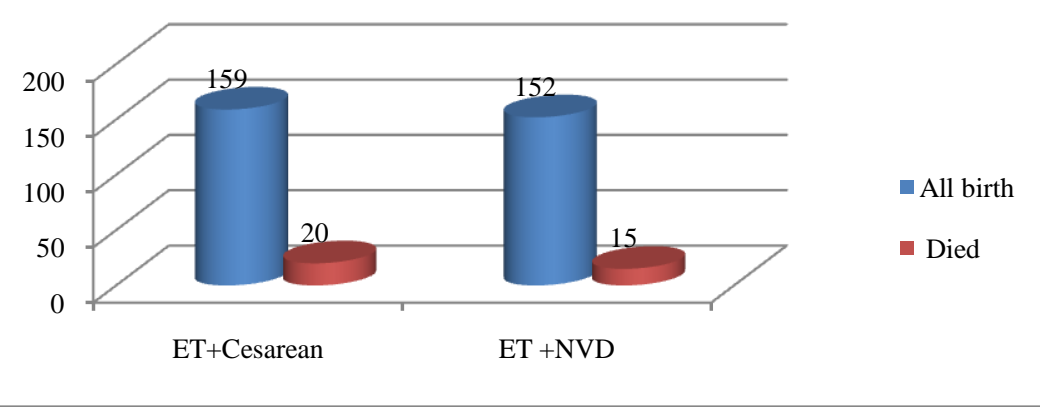


A Study Of Causes And Rate Of Neonatal Mortality In A Teritiary Care Teaching...

Table 8. Neonatal deaths in early term based on Mode of delivery

\begin{tabular}{|c|l|l|l|l|l|l|}
\hline TERMS & All birth & Died & Survived & $\begin{array}{c}\text { P } \\
\text { value }\end{array}$ & Relative risk & $\begin{array}{c}95 \% \\
\text { CI }\end{array}$ \\
\hline ET+ LSCS & & & & & & \\
YES & 159 & 20 & 139 & 0.4742 & 1.317 & 0.6996, \\
NO & 157 & 15 & 142 & & & 2.478 \\
\hline ET + NVD & & & 137 & 0.5918 & 0.8092 & 0.4300, \\
YES & 152 & 15 & 147 & & & 1.523 \\
NO & 164 & 20 & 144 &
\end{tabular}

Gestational age:

Table 9. Showing Gestational age classification.

\begin{tabular}{|c|c|}
\hline Classification & Gestational age in weeks \\
\hline Preterm & $<37$ weeks \\
\hline Early term & $37-38$ weeks \\
\hline Full term & $39-40$ weeks \\
\hline Late term & 41 weeks \\
\hline Post term & $>=42$ weeks \\
\hline
\end{tabular}

1009 Neonates were born at term. Of 489 preterm $106(21.5 \%)$ died, 320 terms babies 35(11.1\%) died and of 200 full term babies $34(17 \%)$ died. 6 were born at post term in which $2(33.3 \%)$ died. No neonatal admissions and deaths occurred at late term (Table10, Figure 7).

Table 10. Neonatal deaths based on Gestational age

\begin{tabular}{|c|c|c|c|}
\hline Terms & All births & Died (n) & $\%$ \\
\hline Preterm & 489 & 106 & 21.5 \\
\hline Early term & 320 & 35 & 11.1 \\
\hline Full term & 200 & 34 & 17 \\
\hline Post term & 6 & 2 & 33.3 \\
\hline
\end{tabular}

Figure 7. Showing the Neonatal deaths based on Gestational age

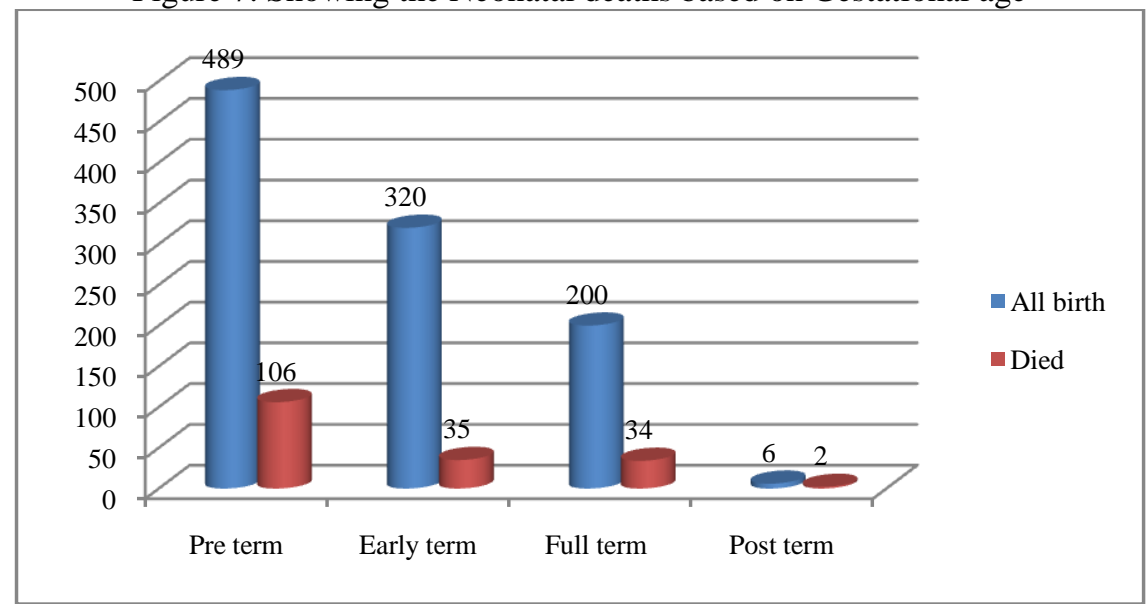

Table 11. Gestational age wise neonatal deaths

\begin{tabular}{|c|c|c|c|c|c|c|}
\hline TERMS & All birth & Died & Survived & $\mathrm{P}$ value & Relative risk & $95 \% \mathrm{CI}$ \\
\hline $\begin{array}{c}\text { PRE TERM } \\
\text { YES } \\
\text { NO }\end{array}$ & $\begin{array}{l}489 \\
529 \\
\end{array}$ & $\begin{array}{l}106 \\
79\end{array}$ & $\begin{array}{l}383 \\
450 \\
\end{array}$ & 0.0057 & 1.452 & $1.115,1.890$ \\
\hline $\begin{array}{c}\text { EARLY TERM } \\
\text { YES } \\
\text { NO } \\
\end{array}$ & $\begin{array}{l}320 \\
698 \\
\end{array}$ & $\begin{array}{l}35 \\
150 \\
\end{array}$ & $\begin{array}{l}285 \\
548 \\
\end{array}$ & $<0.0001$ & 0.5090 & $0.3610,0.7175$ \\
\hline $\begin{array}{c}\text { FULL TERM } \\
\text { YES } \\
\text { NO } \\
\end{array}$ & $\begin{array}{l}200 \\
818 \\
\end{array}$ & $\begin{array}{l}34 \\
151 \\
\end{array}$ & $\begin{array}{l}166 \\
667 \\
\end{array}$ & 0.6831 & 0.9209 & $0.6565,1.292$ \\
\hline $\begin{array}{c}\text { POST TERM } \\
\text { YES } \\
\text { NO }\end{array}$ & $\begin{array}{l}6 \\
1012 \\
\end{array}$ & $\begin{array}{l}2 \\
183 \\
\end{array}$ & $\begin{array}{l}4 \\
829\end{array}$ & 0.2997 & 1.843 & $0.5899,5.761$ \\
\hline
\end{tabular}

\section{Causes Of Death}

The main causes of neonatal deaths were low birth weight, sepsis, respiratory distress, hypoxic ischemic encephalopathy, congenital abnormalities. 
Low Birth Weight (LBW) had highest attributable causes of death. Out of total 484 LBW babies admitted, 115 deaths were reported i.e. LBW accounts $62.16 \%$ of total deaths $(\mathrm{RR}=1.813 ; \mathrm{CI}=1.383-2.376 ; \mathrm{P}=$ $<0.0001)$. Out of total 470 Sepsis babies admitted, 101 deaths were reported i.e. sepsis accounts $54.59 \%$ of total deaths $(\mathrm{RR}=1.402 ; \mathrm{CI}=1.079-1.822 ; \mathrm{P}=0.0116)$.

Out of total 407 Respiratory distress babies admitted, 96 deaths were reported i.e. RDS accounts for $51.89 \%$ of total deaths $(\mathrm{RR}=1.619 ; \mathrm{CI}=1.249-2.100 ; \mathrm{P}=0.0003)$.

Out of total 182 HIE babies admitted 41 deaths were reported i.e. HIE accounts $22.16 \%$ of total deaths $(R R=$ 1.308; $\mathrm{CI}=0.9614-1.779 ; \mathrm{P}=0.1110)$.

Out of total 95 Congenital abnormalities babies admitted, 28 deaths were reported i.e. Congenital abnormalities accounts $15.135 \%$ of total deaths $(\mathrm{RR}=1.733 ; \mathrm{CI}=1.231-2.440 ; \mathrm{P}=0.0048)$.

Out of total 219 babies admitted with other reasons, 47 deaths were reported accounts for $25.41 \%$ of total deaths $(\mathrm{RR}=1.243 ; \mathrm{CI}=0.9248-1.670 ; \mathrm{P}=0.1662)$. These reasons include syndrome babies, respiratory failure, hypothermia, hyperthermia (Table12 Figure 8).

Table12. Causes of Neonatal deaths

\begin{tabular}{|l|c|c|c|}
\hline Death reasons & All births & Deaths (n) & \% \\
\hline Low birth weight (LBW) & 484 & 115 & 23.8 \\
\hline Sepsis & 470 & 101 & 21.5 \\
\hline Respiratory distress (RD) & 407 & 96 & 23.6 \\
\hline $\begin{array}{l}\text { Hypoxic ischemic } \\
\text { encephalopathy( HIE) }\end{array}$ & 182 & 41 & 22.5 \\
\hline Congenital abnormalities (CA) & 95 & 28 & 29.5 \\
\hline Others & 219 & 47 & 21.5 \\
\hline
\end{tabular}

Figure8. Showing the Causes of Neonatal deaths

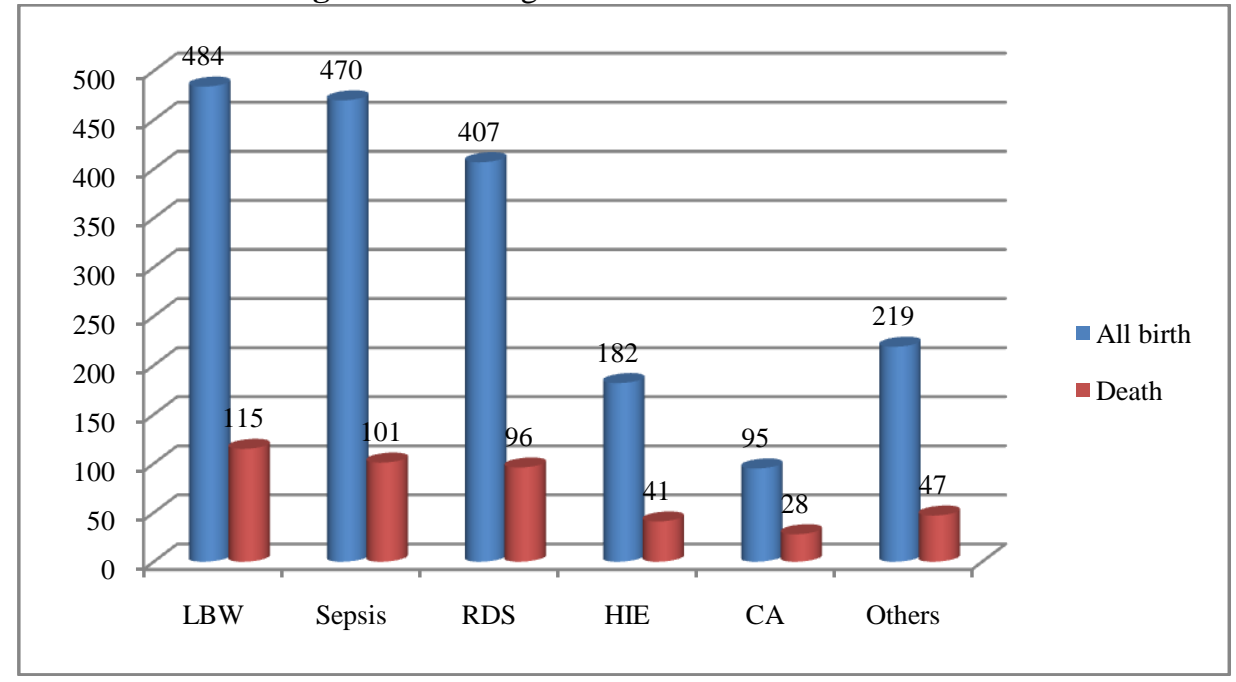

Table13. Major causes of neonatal deaths

\begin{tabular}{|c|c|c|c|c|c|c|}
\hline Death reason & All birth & Died & Survived & $\mathrm{P}$ value & Relative risk & $95 \% \mathrm{CI}$ \\
\hline $\begin{array}{ll}\text { LBW } & \\
& \text { YES } \\
& \text { NO }\end{array}$ & $\begin{array}{l}484 \\
534\end{array}$ & $\begin{array}{l}115 \\
70\end{array}$ & $\begin{array}{l}369 \\
464\end{array}$ & $<0.0001$ & 1.813 & $1.383,2.376$ \\
\hline $\begin{array}{c}\text { Sepsis } \\
\text { YES } \\
\text { NO }\end{array}$ & $\begin{array}{l}470 \\
545 \\
\end{array}$ & $\begin{array}{l}101 \\
84 \\
\end{array}$ & $\begin{array}{l}369 \\
464 \\
\end{array}$ & 0.0116 & 1.402 & $1.079,1.822$ \\
\hline $\begin{array}{ll}\text { RDS } & \\
& \text { YES } \\
& \text { NO } \\
\end{array}$ & $\begin{array}{l}407 \\
611 \\
\end{array}$ & $\begin{array}{l}96 \\
89 \\
\end{array}$ & $\begin{array}{l}311 \\
522 \\
\end{array}$ & 0.0003 & 1.619 & $1.249,2.100$ \\
\hline $\begin{array}{cc}\text { HIE } & \\
& \text { YES } \\
& \text { NO }\end{array}$ & $\begin{array}{l}182 \\
836 \\
\end{array}$ & $\begin{array}{l}41 \\
144\end{array}$ & $\begin{array}{l}141 \\
692 \\
\end{array}$ & 0.1110 & 1.308 & $0.9614,1.779$ \\
\hline $\begin{array}{r}\text { Congenital } \\
\text { YES } \\
\mathrm{NO} \\
\end{array}$ & $\begin{array}{l}95 \\
923\end{array}$ & $\begin{array}{l}28 \\
157 \\
\end{array}$ & $\begin{array}{l}67 \\
766 \\
\end{array}$ & 0.0048 & 1.733 & $1.231,2.440$ \\
\hline $\begin{array}{l}\text { Others } \\
\text { YES } \\
\text { NO }\end{array}$ & $\begin{array}{l}219 \\
799 \\
\end{array}$ & $\begin{array}{l}47 \\
138 \\
\end{array}$ & $\begin{array}{l}172 \\
661 \\
\end{array}$ & 0.1662 & 1.243 & $0.9248,1.670$ \\
\hline
\end{tabular}




\section{Associated causes of deaths:}

Out of total 31 babies with Birth asphyxia admitted, 12 babies deaths were reported i.e. it accounts $6.49 \%$ of total deaths $(\mathrm{RR}=2.208 ; \mathrm{CI}=1.390-3.510 ; \mathrm{P}=0.0070)$.

Out of total 31 babies admitted with Meconium aspiration, 10 deaths were reported i.e. it accounts $5.405 \%$ of total deaths $(\mathrm{RR}=1.819 ; \mathrm{CI}=1.073-3.084 ; \mathrm{P}=0.0548)$.

Out of total 54 babies with Meconium stained liquor admitted 7 babies deaths were reported i.e. it accounts $3.783 \%$ of total deaths $(\mathrm{RR}=0.7020 ; \mathrm{CI}=0.3473-1.1419 ; \mathrm{P}=0.3678)$.

Out of total 196 babies admitted, with Jaundice, 6 deaths were reported i.e. it accounts 3.243\%of total deaths $(\mathrm{RR}=0.1406 ; \mathrm{ci}=0.06325-0.3124 ; \mathrm{P}=0.0001)$.

Out of 36 babies admitted with Neonatal convulsions, 6babies death were reported i.e. it accounts $3.243 \%$ of total deaths $(\mathrm{RR}=0.9143 ; \mathrm{CI}=0.4351-1.921 ; \mathrm{P}=1.00)$ (Table16, figure9).

Table14. Associated causes of Neonatal deaths

\begin{tabular}{|l|c|c|c|}
\hline Death reasons & All births & Deaths (n) & $\%$ \\
\hline Birth asphyxia (BA) & 31 & 12 & 38.7 \\
\hline Meconium aspiration(MAS) & 31 & 10 & 32.2 \\
\hline Meconium stained liquor(MSL) & 54 & 7 & 12.9 \\
\hline Neonatal jaundice( NNJ) & 196 & 6 & 3.1 \\
\hline Neonatal convulsions (NNC) & 36 & 6 & 16.7 \\
\hline
\end{tabular}

Figure 9. Showing the Associated causes of Neonatal deaths

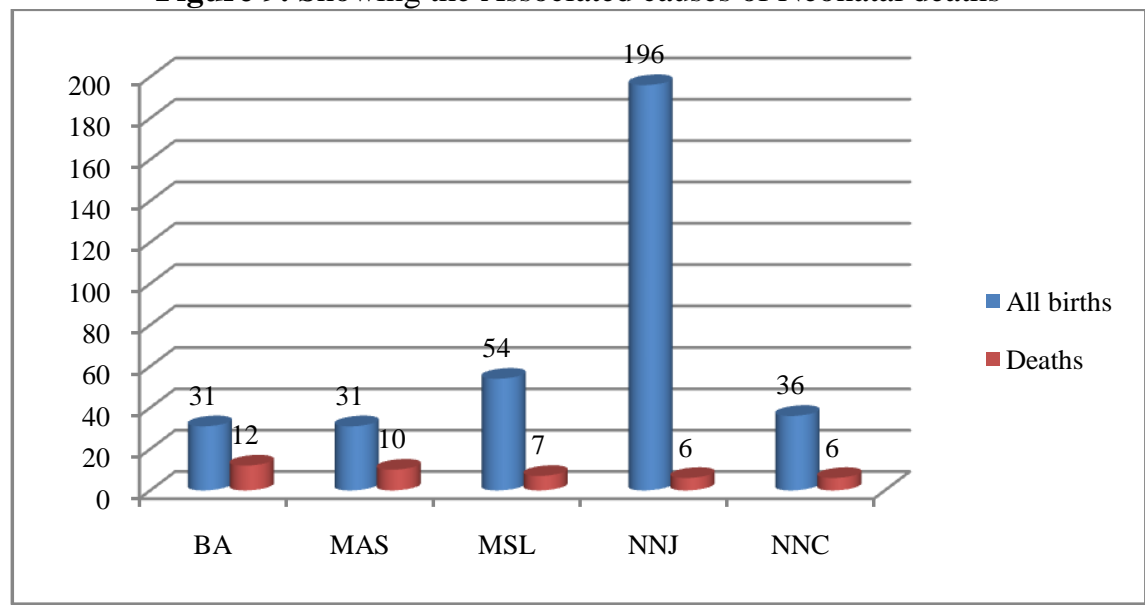

Table15. ASSOCIATED CAUSES OF DEATH

\begin{tabular}{|c|c|c|c|c|c|c|}
\hline Death reason & All birth & Died & Survived & $\mathrm{P}$ value & $\begin{array}{l}\text { Relative } \\
\text { risk }\end{array}$ & $95 \% \mathrm{CI}$ \\
\hline $\begin{array}{c}\text { Birth asphyxia } \\
\text { YES } \\
\text { NO }\end{array}$ & $\begin{array}{c}31 \\
987\end{array}$ & $\begin{array}{c}12 \\
173\end{array}$ & $\begin{array}{c}19 \\
814\end{array}$ & 0.0070 & 2.208 & $1.390,3.510$ \\
\hline $\begin{array}{l}\text { MAS } \\
\text { YES } \\
\text { NO }\end{array}$ & $\begin{array}{c}31 \\
987\end{array}$ & $\begin{array}{c}10 \\
175 \\
\end{array}$ & $\begin{array}{c}21 \\
812 \\
\end{array}$ & 0.0548 & 1.819 & $1.073,3.084$ \\
\hline $\begin{array}{l}\text { MSL } \\
\text { YES } \\
\text { NO } \\
\end{array}$ & $\begin{array}{c}54 \\
964 \\
\end{array}$ & $\begin{array}{c}7 \\
178 \\
\end{array}$ & $\begin{array}{c}47 \\
786 \\
\end{array}$ & 0.3678 & 0.7020 & $0.3473,1.419$ \\
\hline $\begin{array}{c}\text { Jaundice } \\
\text { YES } \\
\text { NO }\end{array}$ & $\begin{array}{l}196 \\
822\end{array}$ & $\begin{array}{c}6 \\
179 \\
\end{array}$ & $\begin{array}{l}190 \\
643 \\
\end{array}$ & 0.0001 & 0.1406 & $0.06325,0.3124$ \\
\hline $\begin{array}{l}\text { Seizures } \\
\text { YES } \\
\text { NO }\end{array}$ & $\begin{array}{c}36 \\
982\end{array}$ & $\begin{array}{c}6 \\
179\end{array}$ & $\begin{array}{c}30 \\
803\end{array}$ & 1.000 & 0.9143 & $0.4351,1.921$ \\
\hline
\end{tabular}

In lbw neonates:

Major causes of deaths include sepsis $(87.8 \%)$, RD (83.5\%), HIE (35.7\%), Congenital abnormalities $(24.3 \%)$ (Table 16, Figure10).

Gestational age wise deaths include preterm (87.8\%), term (12.2\%) (Table14, Figure 10). 
Table16. deaths based on neonatal characteristics

\begin{tabular}{|c|c|c|c|c|}
\hline Characteristics & Died & Survived & Chi square & P value \\
\hline GENDER & 111 & 478 & 0.4252 & 0.8085 \\
Males & 74 & 355 & & \\
Females & 185 & 832 & & \\
Total & & & & \\
\hline GESTATIONAL AGE & 106 & 383 & 13.43 & 0.0012 \\
Pre term (<37) & 69 & 451 & & \\
Term (37-41) & 2 & 4 & & \\
Post term(>=42) & 20 & 2 & 116.8 & \\
\hline BIRTH WEIGHT & 29 & 38 & & \\
$<1$ & 66 & 330 & & \\
$1-1.49$ & 69 & 460 & & \\
$1.5-2.49$ & 1 & 3 & & \\
$2.5-3.99$ & & & & \\
$>/ 4.0$ & & & & \\
& & & & \\
\end{tabular}

Table17. Death reasons in LBW neonates (Total deaths in LBW 115)

\begin{tabular}{|c|l|l|l|l|}
\hline Reason for Death & Total births & Total Survival & Total Deaths $(\mathrm{n})$ & Death \%in LBW \\
\hline Sepsis & 242 & 141 & 101 & 87.8 \\
\hline RD & 224 & 128 & 96 & 83.5 \\
\hline HIE & 64 & 23 & 41 & 35.7 \\
\hline CA & 33 & 5 & 28 & 24.3 \\
\hline Others & 136 & 85 & 51 & 44.3 \\
\hline
\end{tabular}

Figure10. Showing the Deaths reasons in LBW neonates

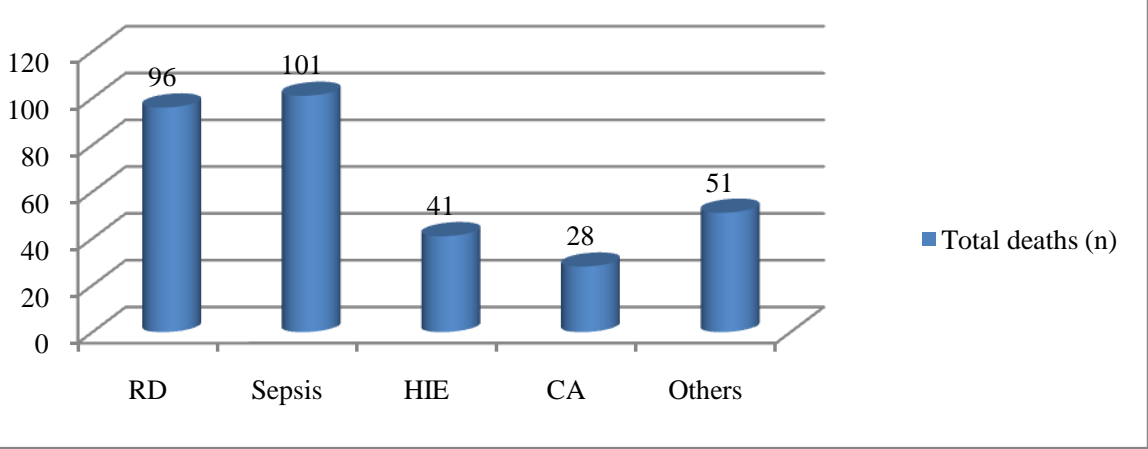

Table18. Gestational age wise deaths in LBW neonates

\begin{tabular}{|c|l|l|l|l|}
\hline Gestational age & Total births & Total Survival & Total Deaths $(\mathrm{n})$ & \%Of deaths \\
\hline Pre term & 378 & 278 & 101 & 87.8 \\
\hline Term (ET+ FT) & 105 & 91 & 14 & 12.2 \\
\hline Post term & 1 & 1 & 0 & 0 \\
\hline
\end{tabular}

Figure11. Showing Gestational age wise deaths LBW neonates

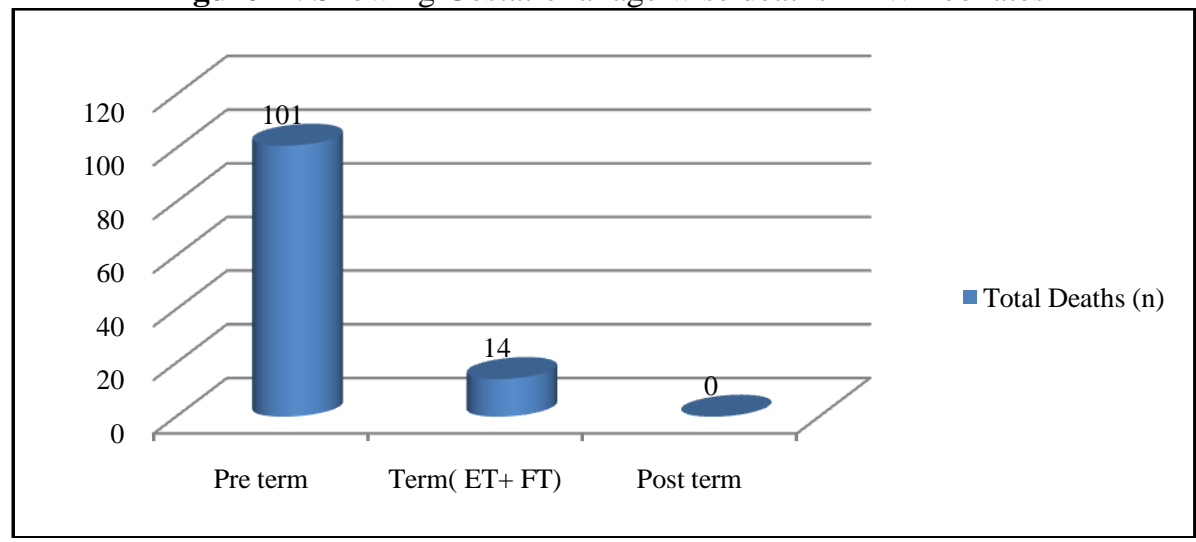

IV. Discussion

The study of mortality risk measurement among the new born admitted to the neonatal intensive care units is attaining an increasing level of importance. This study has determined the burden and associated with neonatal mortality. The neonatal mortality rate was 18.17 per 100 live births which are 6 times more when 
compared to study by Anthony O. Adetola et al ${ }^{[1]}$ they have reported the NMR was 3.21 per 100 live births male babies have a higher risk than females of dying during the neonatal period. $81.62 \%$ of deaths accounts for rural area admissions hence more care should be taken for rural area admissions. High neonatal deaths (74.6\%) have been reported at early days of life (0-6). Preterm birth is generally attributed as a direct cause of death while LBW is considered as a risk factor or underlying condition. Cesarean deliveries in early term are more when compared to Normal and Assisted vaginal deliveries in early term. Major causes of deaths include LBW (62.16\%) followed by sepsis $(54.59 \%)$, RD (51.89\%), HIE (22.16\%) and Congenital abnormalities $(15.13 \%)$. In LBW major causes of death include Sepsis (87.8\%) followed by RD (83.5\%) and preterm deaths (87.8\%) are more when compared to term gestation (12.27\%). And major associated causes of the deaths include Birth asphyxia followed by Meconium aspiration, Meconium stained liquor, Neonatal jaundice and Neonatal convulsions. Here in our study sepsis includes both viral and bacterial infections of various etiologies. No standard definition for birth asphyxia exists, particularly at community level, and remedying of this situation has been identified as a research priority. More than fifty percent of deaths occurred at preterm. Early initiation of breast feeding may reduce neonatal mortality by decreasing the ingestion of infectious pathogens. For unknown babies we did not get the maternal information which includes mode of delivery, gestational age, antenatal care, previous illness to mothers and drug use. Silverman retraction score, Downs score, Ballard scores also collected but the data was not available for all neonates hence their significance in study was not mentioned. As with previous studies, it was observed that breech presentation had 5 times higher risk of neonatal death compared with cephalic presentation but here in our study due to lack of data and information from patients attendants this was not clearly known.

\section{Conclusion}

Improvement in the neonatal resuscitation skills in this environment is evident as RD, asphyxia continued to be leading cause of death with basic neonatal resuscitation kills, up to $90 \%$ of the babies who die because of asphyxia might be saved. Instrapartum problems, such as meconium-stained or foul-smelling liquor, and meconium aspiration were factors that increased the risk of neonatal deaths. These factors predispose the newborn to significant risk of infection. Clean delivery may be necessary but not sufficient to reduce neonatal mortality. Innovative approaches to improve access to essential health services for neonatal complications Should be tried within the existing health systems to ensure sustainability. Further much work is needed to educate community members about neonatal danger signs and to engage them in effective care-seeking behavior .The process of delivery should be made safe for the mother and neonate. We found LBW, Sepsis and RD to be the major cause's neonatal death.

[1]. Anthony O, Adetola, Olukemi O.Tongo, Adetola E. Orimadegun, Kike Osinusi. Neonatal Mortality in an Urban Population in India, Nigeria. Pediatrics and Neonatology. 2011; 52:243-250

[2]. The UN Inter-agency Group for Child Mortality Estimation. Levels and Trends in ChildMortality,1990.2010.2011.http://www.healthynewbornnetwork.org/resource/levelsand-trends-child-mortality-2011-report-acc essed 1 September 2012.

[3]. Oestergaard MZ, Inoue M,Yoshida S, Mahanani WR, Gore FM, Cousens S, et al. Neonatal mortality levels for 193 countries in 2009 with trends since 1990: A systematic analysis of progress, projections, and priorities. PloS Medicine. 2011; 8(8): e1001080.

[4]. Pavitra Mohan, Baya Kishore, Sharad Singh, Rajiv Bahi, Anju Puri, Rajesh Kumar. Assessment of Implementation of Integrated Management of Neonatal and Childhood Illness in India. J Health Popul Nutr. 2011 Dec; 29(6):629-38.

[5]. Tasneem Kousar, Yasmeen Memon, Salma Sheik, Shazia Memon, Rubina Sehto. Risk factors and causes of death in Neonates. Rawal Medical Journal. 2010; 35(2):205-208.

[6]. AH Baqui, GL Darmstadt, EK Williams, et al. Rates, timing and causes of neonatal deaths in rural India: implications for neonatal health programmes. Bulletin of the World Health Organization 2006; 84:706-713.

[7]. Fatema Khatun, Sabrina Rasheed, Allisyn C Moran, et al. causes of neonatal and maternal deaths in Dhaka Slums Implications for service delivery. BMC Public Health 2012; 12:84.

[8]. Diego G Bassani, Rajesh Kumar,Shally Awashi, et al. Causes of maternal and child mortality: a nationally representative mortality survey. Lancet 2010; 376:1853-60.

[9]. Lawn, JE, S.Cousens, and J. Zupan. 4 million neonatal deaths: when? Where? Why? Lancet, 2005; 365(9462): 891-900.

[10]. Acuin CS, Khor GL, Liabsuetrakul T, Achadi EL, Htay TT, Firestone R, et al. Maernal, neonatal, and child health in Southeast Asia: towards greater regional collaboration. Lancet. 2011; 377(9764): 516-25.

[11]. Robert L Goldenberg, Jennifer F Culhane. Low birth weight in the United States. Am J Clin Nutr 2007; 85(suppl): 584S-90S

[12]. International classification of diseases and related health problems. $10^{\text {th }}$ revision. Geneva: World Health Organization; 1992.

[13]. Huddy CL, Johnson A, Hope PL. Educational and behavioral problems in babies of 32-35 weeks gestation. Arch Dis Child Fetal Neonatal Ed 2001; 85: 23F-8.

[14]. Lawn JE, Wilczynska-Ketende K, Cousens SN. Estimating the causes of 4 million neonatal deaths in the year 2000. Int J Epidemiol 2006; 35: 706-18.

[15]. Lawn JE, Cousens SN, Darmstadt GL, Bhutta ZA, Martines J, Paul V, et al. 1 year after The Lancet Neonatal Survival Series-was the call for action heard. Lancet 2006; 367: 1541-7.

[16]. P07 - Disorders related to short gestation and low birth weight in ICD-10

[17]. eMedicine-Extremely low birth weight infant: article by KN Siva Subramanian,MD. http://emedicine.medscape.com/article/979717-overview. 